\title{
Circumscribing Polygons and Polygonizations for Disjoint Line Segments
}

\author{
Hugo A. Akitaya
}

Department of Computer Science, Tufts University, Medford, MA, USA

hugo.alves_akitaya@tufts.edu

\section{Matias Korman}

Department of Computer Science, Tufts University, Medford, MA, USA

matias.korman@tufts.edu

\section{Mikhail Rudoy}

CSAIL, Massachusetts Institute of Technology, Cambridge, MA, USA

Google Inc., Cambridge, MA, USA

mrudoy@gmail.com

\section{Diane L. Souvaine}

Department of Computer Science, Tufts University, Medford, MA, USA

diane.souvaine@tufts.edu

\section{Csaba D. Tóth}

Department of Mathematics, California State University Northridge, Los Angeles, CA Department of Computer Science, Tufts University, Medford, MA, USA

csaba.toth@csun.edu

\begin{abstract}
Given a planar straight-line graph $G=(V, E)$ in $\mathbb{R}^{2}$, a circumscribing polygon of $G$ is a simple polygon $P$ whose vertex set is $V$, and every edge in $E$ is either an edge or an internal diagonal of $P$. A circumscribing polygon is a polygonization for $G$ if every edge in $E$ is an edge of $P$.

We prove that every arrangement of $n$ disjoint line segments in the plane has a subset of size $\Omega(\sqrt{n})$ that admits a circumscribing polygon, which is the first improvement on this bound in 20 years. We explore relations between circumscribing polygons and other problems in combinatorial geometry, and generalizations to $\mathbb{R}^{3}$.

We show that it is NP-complete to decide whether a given graph $G$ admits a circumscribing polygon, even if $G$ is 2-regular. Settling a 30-year old conjecture by Rappaport, we also show that it is NP-complete to determine whether a geometric matching admits a polygonization.
\end{abstract}

2012 ACM Subject Classification Theory of computation $\rightarrow$ Computational geometry; Mathematics of computing $\rightarrow$ Combinatoric problems

Keywords and phrases circumscribing polygon, Hamiltonicity, extremal combinatorics

Digital Object Identifier 10.4230/LIPIcs.SoCG.2019.9

Related Version A full version of the paper is available on arXiv [2], http://arxiv.org/abs/1903. 07019.

Funding Research supported in part by the NSF awards CCF-1422311 and CCF-1423615.

Matias Korman: Partially supported by MEXT KAKENHI No. 17K12635.

Diane L. Souvaine: Partially supported by the Erwin Schrödinger Institute for Mathematics and Physics (ESI).

(c) (i) () H. A. Akitaya, M. Korman, M. Rudoy, C. D. Tóth, and D. L. Souvaine;

cc. licensed under Creative Commons License CC-BY

35th International Symposium on Computational Geometry (SoCG 2019).

Editors: Gill Barequet and Yusu Wang; Article No. 9; pp. 9:1-9:17

Leibniz International Proceedings in Informatics 


\section{Introduction}

Reconstruction of geometric objects from partial information is a classical problem in computational geometry. In this paper, we revisit the problem of reconstructing a simple polygon (alternatively, a triangulated simple polygon) $P$ when some of its edges have been lost. Given a set $V$ of $n$ points in the plane, a polygonization of $V$ is a simple polygon $P$ whose vertex set is $V$. It is easy to see that, unless all points are collinear, $V$ has a simple polygonization. The number of polygonizations is exponential in $n$, and there is extensive work on determining the minimum and maximum number of polygonizations for $n$ points in general position as a function of $n$ (see [6] and [20] for the latest upper and lower bounds, and [9] for a survey on this and related problems).

A natural generalization of this problem is to augment a given planar straight-line graph (PSLG) $G=(V, E)$ into a simple polygon or a Hamiltonian PSLG. In particular, three variants have been considered: A simple polygon $P$ on a vertex set $V$ is a polygonization if every edge in $E$ is an edge of $P$; a circumscribing polygon if every edge in $E$ is an edge or an internal diagonal in $P$; and a compatible Hamiltonian polygon if every edge in $E$ is an edge, an internal diagonal, or an external diagonal in $P$.

Hoffmann and Tóth [8] proved that every planar straight-line matching admits a compatible Hamiltonian polygon, unless all segments are collinear, in which case no such polygon exists. Urabe and Watanabe [21] constructed an arrangement of 16 disjoint segments that does not admit a circumscribing polygon. However, a circumscribing polygon is known to exist when (i) each segment has at least one endpoint on the boundary of the convex hull [13], or (ii) no segment intersects the supporting line of any other segment [14]. Pach and Rivera-Campo [16] proved in 1998 that every set of $n$ disjoint segments contains a subset of $\Omega\left(n^{1 / 3}\right)$ segments that admits a circumscribing polygon; no nontrivial upper bound is known.

Rappaport [18] proved that it is NP-complete to decide whether $G$ can be augmented into a simple polygon. In the reduction, $G$ consists of disjoint paths, and Rappaport conjectured that the problem remains hard even if $G$ is a perfect matching (i.e., disjoint line segments in the plane). In the special case that $\mathrm{G}$ is perfect matching and every segment has at least one endpoint on the boundary of the convex hull, then an $O(n \log n)$ time algorithm can compute a polygonization (or report that none exists [19]). If $S$ is a set of $n \geq 3$ parallel chords of a circle, then neither $S$ nor any subset of 3 or more segments from $S$ admits a polygonization (so the analogue of the problem of Pach and Rivera-Campo [16] has a trivial answer in this case). In a related result, Ishaque et al. [10] proved that $n$ disjoint line segments in general position, where $n$ is even, can be augmented to a 2-regular PSLG (i.e., a union of disjoint simple polygons).

Our Results. In this paper, we obtain the following results.

- We prove that every set of $n$ disjoint line segments in general position contains a subset of $\Omega(\sqrt{n})$ segments that admit a circumscribing polygon (Theorem 1 in Section 2). This is the first improvement over the previous bound of $\Omega\left(n^{1 / 3}\right)[16]$ in the last 20 years.

- While we do not have any nontrivial upper bound for circumscribing polygons proper, we relate that problem to the extensibility of disjoint line segments to disjoint rays. For every $n \in \mathbb{N}$, we construct a set of $n$ disjoint line segments in the plane such that the size of any subset extensible to disjoint rays is $O(\sqrt{n})$ (Section 3 ).

- We prove that it is NP-complete to determine whether a given set of disjoint cycles in the plane admits a circumscribing polygon (Theorem 13 in Section 4). The reduction is from Hamiltonian paths in 3-connected cubic planar graphs.

- We prove that it is NP-complete to determine whether a given set of disjoint line segments admits a polygonization (Theorem 14 in Section 5). This settles a 30-year old conjecture by Rappaport [18] in the affirmative. 
We conclude with a few open problems and three-dimensional generalizations in Section 6 . All omitted proofs are available in the full version of this paper [2].

Further Related Previous Work. Hamiltonicity fascinated graph theorists and geometers for centuries. Some planar graph results hold for PSLGs, as well (i.e., planar graphs with a fixed straight-line embeddings). Hamiltonicity is NP-complete for planar cubic graphs [7], but can be solved in linear time in 4-connected planar graphs [4], and all 4-connected triangulations (i.e., edge-maximal planar graphs) are Hamiltonian [22]. In terms of augmentation, a nonHamiltonian triangulation cannot be augmented to a Hamiltonian planar graph by adding edges or vertices. However, Pach and Wenger [17] proved that every planar graph on $n$ vertices can be transformed into a Hamiltonian planar graph on at most $5 n$ vertices by subdividing some of the edges, with at most two new vertices per edge, and by adding new edges. See also the surveys [5, 15] on Hamiltonicity of planar graphs and their applications.

\section{Large Subsets with Circumscribing Polygons}

For every integer $n \geq 2$, let $f(n)$ be the maximum integer such that every set of $n$ disjoint segments in the plane in general position contains a subset of $f(n)$ segments that admit a circumscribing polygon. Pach and Rivera-Campo [16] proved 20 years ago that $f(n)=$ $\Omega\left(n^{1 / 3}\right)$. In this section, we improve the lower bound to $f(n)=\Omega(\sqrt{n})$.

- Theorem 1. Every set of $n \geq 2$ disjoint line segments in the plane in general position contains $\Omega(\sqrt{n})$ segments that admit a circumscribing polygon.

Proof. Segment Selection. Let $S$ be a set of $n \geq 2$ disjoint line segments in the plane. We may assume without loss of generality that none of the segments is vertical, and all segment endpoints have distinct $x$-coordinates. For a subset $S^{\prime} \subseteq S$, a halving line is a vertical line $\ell$ such that the number of segments in $S^{\prime}$ contained in the left and right open halfplanes bounded by $\ell$ differ by at most one. In particular, each halfplane contains at most $\left|S^{\prime}\right| / 2$ segments from $S^{\prime}$.

We partition $S$ recursively as follows. Find a halving line $\ell$ for $S$, and recurse on the nonempty subsets of segments lying in each open halfplane determined by $\ell$. Denote by $T$ the recursion tree, which is a binary tree of depth at most $\log n$. We denote by $V(T)$ the set of nodes of $T$, and by $V_{i}(T)$ the set of nodes at level $i$ of $T$ for $i=0,1, \ldots,\lfloor\log n\rfloor$. Associate each node $v \in V(T)$ to a halving line $\ell_{v}$ and to the subset $S_{v} \subseteq S$ of segments that intersect $\ell_{v}$ without intersecting the halving lines associated with any ancestor of $v$. This defines a partition of $S$ into subsets $S_{v}, v \in V(T)$.

For every $v \in V(T)$, sort the segments in $S_{v}$ by the $y$-coordinates of their intersections with the line $\ell_{v}$; and let $Q_{v} \subseteq S_{v}$ be a maximum subset of segments that have monotonically increasing or decreasing slopes. By the Erdős-Szkeres theorem, we have $\left|Q_{v}\right| \geq \sqrt{\left|S_{v}\right|}$ for every $v \in V(T)$. For a refined analysis, we consider the union of the sets $Q_{v}$ for $v \in V_{i}(T)$ for $i=0, \ldots,\lfloor\log n\rfloor$, and then take one such union of maximal cardinality.

We need some additional notation. For every $v \in V(T)$, let $n_{v}=\left|S_{v}\right|$ and $m_{v}=\left|Q_{v}\right|$. For every integer $i=0,1, \ldots,\lfloor\log n\rfloor$, let $\mathcal{S}_{i}$ (resp., $\mathcal{Q}_{i}$ ) be the union of $S_{v}$ (resp., $Q_{v}$ ) over all vertices $v \in V_{i}(T)$. Let $\nu_{i}=\left|\mathcal{S}_{i}\right|$ and $\mu_{i}=\left|\mathcal{Q}_{i}\right|$. By definition, we have $n=\sum_{i=0}^{\lfloor\log n\rfloor} \nu_{i}$.

Let $M=\max \left\{\mu_{i}: 0 \leq i \leq\lfloor\log n\rfloor\right\}$. We claim that

$M \geq \sqrt{n} / 2$. 
By the Erdös-Szekeres Theorem, we have $m_{v} \geq \sqrt{n_{v}}$ for every $v \in V(T)$. Since $n_{v} \leq n / 2^{i}$ for every $v \in V_{i}(T)$, then $m_{v} \geq \sqrt{n_{v}}=n_{v} / \sqrt{n_{v}} \geq n_{v} / \sqrt{n / 2^{i}}=\sqrt{2^{i} / n} \cdot n_{v}$. Summation over all $v \in V_{i}(T)$ yields $M \geq \mu_{i} \geq \sqrt{2^{i} / n} \cdot \nu_{i}$, which in turn gives $\nu_{i} \leq M \sqrt{n / 2^{i}}$. Summation over all $i=0, \ldots,\lfloor\log n\rfloor$ now gives $n=\sum_{i=0}^{\lfloor\log n\rfloor} \nu_{i} \leq M \sqrt{n} \sum_{i=0}^{\lfloor\log n\rfloor} 2^{-i / 2} \leq 2 M \sqrt{n}$, hence $M \geq \sqrt{n} / 2$, which proves (1).

Let $i^{*} \in\{0,1, \ldots,\lfloor\log n\rfloor\}$ be an index where $M=\mu_{i^{*}}$, and put $\widehat{S}_{0}=\mathcal{Q}_{i^{*}}$. By construction, $\widehat{S}_{0}=\bigcup\left\{Q_{v}: v \in V_{i^{*}}(T)\right\}$. We further partition $\widehat{S}_{0}$ into two subsets as follows. Let $V_{i^{*}}^{<}$ (resp., $V_{*} i^{>}$) be the set of nodes in $V_{i}(T)$ such that the slopes in $Q_{v}$ monotonically increase (resp., decrease). Let $\widehat{S}_{1}$ be the larger of $\bigcup\left\{Q_{v}: v \in V_{i^{*}}^{<}\right\}$and $\bigcup\left\{Q_{v}: v \in V_{i^{*}}^{>}\right\}$, breaking ties arbitrarily. Note that $\left|\widehat{S}_{1}\right| \geq \sqrt{n} / 4$. We may assume, by a reflection in the $y$-axis if necessary, that $\widehat{S}_{1}=\bigcup\left\{Q_{v}: v \in V_{i^{*}}^{>}\right.$; see Fig. 1 for an example.

Construction of a Circumscribing Polygon. We construct a simple polygon that is a circumscribing polygon for a subset $\widehat{S}_{2} \subseteq \widehat{S}_{1}$ of size $\left|\widehat{S}_{2}\right| \geq\left|\widehat{S}_{1}\right| / 2 \geq \sqrt{n} / 8$. Pach and RiveraCampo [16] proved that an arrangement of disjoint line segments admits a circumscribing polygon if they are (1) stabbed by a vertical line, and (2) have monotonically increasing or decreasing slopes. In particular, each $Q_{v}, v \in V(T)$, admits a circumscribing polygon. In contrast, we construct a circumscribing polygon for at least half of the segments in $\widehat{S}_{1}$, where $\widehat{S}_{1}$ is the union of all $Q_{v}, v \in V_{i^{*}}(T)$, separated by vertical lines.

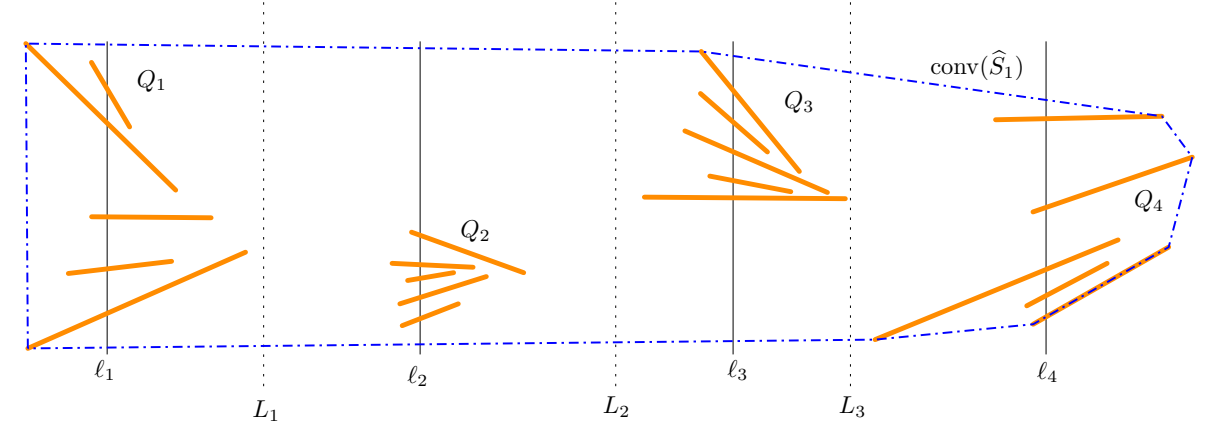

Figure 1 A set $\widehat{S}_{1}=\bigcup\left\{Q_{v}: v \in V_{i^{*}}^{>}\right\}$of 25 line segments for $r=4$; and $P=\operatorname{conv}\left(\widehat{S}_{1}\right)$.

For ease of presentation, we introduce new notation for $\widehat{S}_{1}=\bigcup\left\{Q_{v}: v \in V_{i^{*}}^{<}\right\}$; see Fig. 1 . Denote by $\ell_{1}, \ell_{2}, \ldots, \ell_{r}$ the halving lines $\left\{\ell_{v}: v \in V_{i^{*}}^{<}\right\}$sorted from left-to-right, and let $Q_{1}, Q_{2}, \ldots, Q_{r}$ be the corresponding sets in $\left\{Q_{v}: v \in V_{i^{*}}^{>}\right.$. Denote by $L_{i}(i=1, \ldots, r-1)$ the vertical lines that separate $Q_{i}$ and $Q_{i+1}$. Refer to Fig. 1.

Overview. We construct a circumscribing polygon for a subset $\widehat{S}_{2} \subseteq \widehat{S}_{1}$ incrementally in two phases, while maintaining a polygon in the segment endpoint visibility graph of $\widehat{S}_{1}$. We use a machinery developed in [8], with several important new elements. Initially, let $P$ be the boundary of conv $\left(\widehat{S}_{1}\right)$, which is a simple polygon. Intuitively, think of polygon $P$ as a rubber band, and stretch it successively to visit more segment endpoints from $\widehat{S}_{1}$, maintaining the property that all segments in $\widehat{S}_{1}$ remain in the closed polygonal domain of $P$. A key invariant of $P$ will be that if $P$ visits only one endpoint of some segment in $\widehat{S}_{1}$, then we can stretch it to visit the other endpoint (a strategy previously used in $[8,13]$ ). This tool allows us to produce a circumscribing polygon for a subset of $\widehat{S}_{1}$. It is enough to ensure that $P$ reaches an endpoint of at least half of the segments in $\widehat{S}_{1}$. To do this, we use the fact that each set $Q_{v}, v \in V_{i^{*}}^{>}$, is sorted along the halving lines in decreasing order by slope, and we ensure 
that $P$ reaches the left endpoint of at least half of the segments (later, we stretch $P$ to visit the right endpoints). At the end, we define $\widehat{S}_{2}$ as the set of segments in $\widehat{S}_{1}$ visited by $P$ (i.e., we discard the remaining segments lying in the interior of $P$ ).

We maintain a polygon with the properties listed in Definition 2 below. There are a few important features to note: $P$ is not necessarily a simple polygon in intermediate steps of the algorithm: it is a weakly simple polygon that does not have self-crossings; it has clearly defined interior and exterior; and it can have repeated vertices. Specifically, each vertex can repeat at most twice (i.e., multiplicity at most 2 ), and if its multiplicity is 2 , then one occurrence is a reflex vertex and the other is convex. Furthermore, all such reflex vertices can be removed simultaneously by suitable shortcuts (cf. property (F5) below) to obtain a simple polygon. We need to be very careful about reflex vertices in $P$ : for each reflex vertex in $P$, we ensure either that it will not become a repeated vertex later, or that if it becomes a repeated vertex, then its reflex occurrence can be removed by a suitable shortcut.

Invariants. As in [8], we maintain a weakly simple polygon, called a frame (defined below). A weakly simple polygon is a closed polygonal chain $P=\left(v_{1}, \ldots, v_{k}\right)$ in counterclockwise order such that, for every $\varepsilon>0$, displacing the vertices by at most $\varepsilon$ can produce a simple polygon. Denote by $\widehat{P}$ the union of the interior and the boundary of $P$. A weakly simple polygon may have repeated vertices. Three consecutive vertices $\left(v_{i-1}, v_{i}, v_{i+1}\right)$ define an interior angle $\angle\left(v_{i-1}, v_{i}, v_{i+1}\right)$, or $\angle v_{i}$, which is either convex $\left(\leq 180^{\circ}\right)$ or reflex $\left(>180^{\circ}\right)$.

The following definitions summarizes the properties that we maintain for a polygon $P$. It is based on a similar concept in [8]: we do not allow segments to be external diagonal (cf. (F2)) and relax the conditions on the possible occurrences of reflex vertices. Property (F6) is related to the vertical lines $\ell_{i}(i=1, \ldots, r-1)$ in the instance $S=\bigcup_{i=1}^{r} Q_{i}$. Reflex vertices play an important role. We distinguish two types of reflex vertices: A reflex vertex $v$ of a frame $P$ is safe if the (unique) line segment in $S$ incident to $v$ subdivides the reflex angle $\angle v$ into two convex angles; otherwise $v$ is unsafe.

- Definition 2. A weakly simple polygon $P=\left(v_{1}, \ldots, v_{k}\right)$ is called frame for a set $S$ of disjoint line segments in the plane, if (cf. Fig. 2)

(F1) every vertex of $P$ is an endpoint of some segment in $S$;

(F2) $\widehat{P}$ contains every segment in $S$;

(F3) every vertex in $P$ has multiplicity at most 2;

(F4) if a vertex in $P$ has multiplicity 2, say $v_{i}=v_{j}$, then one of $\angle v_{i}$ or $\angle v_{j}$ is convex (and the other angle is reflex);

(F5) if $\left(v_{i}, \ldots, v_{j}\right)$ is a maximal chain of unsafe reflex vertices of $P$ that each have multiplicity 2, then $\left(v_{j+1}, v_{j}, \ldots v_{i}, v_{i-1}\right)$ is a simple polygon that is interior-disjoint from $P$;

(F6) the vertical line $\ell_{i}(i=1, \ldots, r-1)$ crosses $P$ exactly twice.

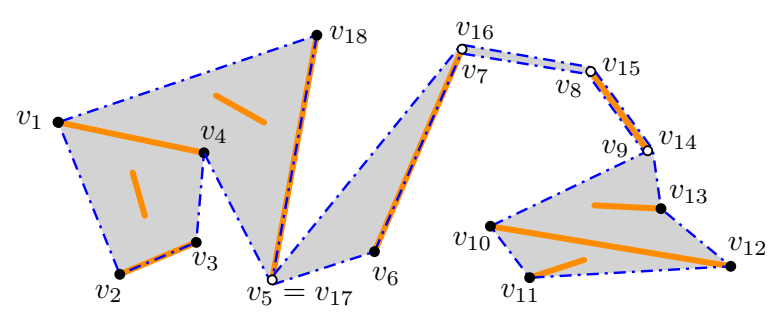

Figure 2 A frame $P=\left(v_{1}, \ldots, v_{18}\right)$ for 10 disjoint line segments (orange). The closed region $\widehat{P}$ is shaded gray. The vertices of multiplicity 1 (resp., 2) are marked with full (resp., empty) dots. 
Elementary Operations. Let $S$ be a set of disjoint line segments in general position, and let $P$ be a frame. We define four elementary operations that each transform $P$ into a new frame for $S$. The first operation is the "shortcut" that eliminates reflex vertices of multiplicity 2 , and increases the area of the interior. The remaining three operations each increase the number of vertices of the frame (possibly creating vertices of multiplicity 2) and decrease the area of its interior.

For shortest path and ray shooting computations, we consider the line segments in $S$ and the current frame $P$ to be obstacles. For a polygonal path $(a, b, c)$ that does not cross any segment in $S$, we define the convex $\operatorname{arc} \operatorname{carc}(a, b, c)$ to be the shortest polygonal path between $a$ and $c$ that is homotopic to $(a, b, c)$.

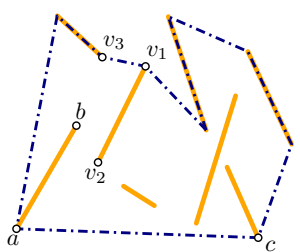

(a)

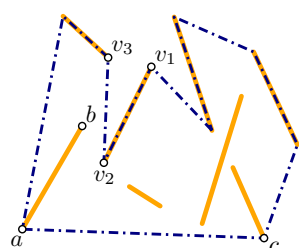

(b)

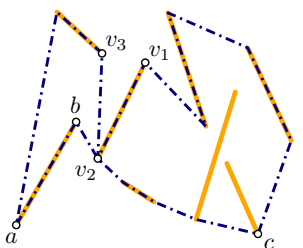

(c)

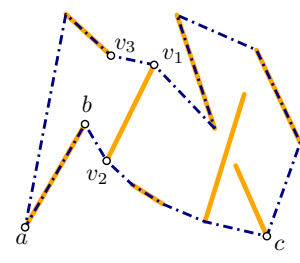

(d)

Figure 3 (a) A frame $P$. (b) $P:=\operatorname{BuildCap}\left(P, 1, v_{1}\right)$. (c) $P:=\operatorname{BuildCap}(P, 1, a)$. (d) $P:=$ ChopWedges $(P)$.

- Operation 1. (ChopWedges $(P)$ ) Refer to Fig. 3(c-d). Input: a frame P. Action: While there is a vertex of multiplicity 2 , do: let $\left(v_{i}, \ldots, v_{j}\right)$ be a maximal chain of convex vertices of $P$ that each have multiplicity 2 , and replace the path $\left(v_{i-1}, v_{i}, \ldots v_{j}, v_{j+1}\right)$ in $P$ by a single edge $v_{i-1} v_{j+1}$.

- Operation 2. (BuildCap $(P, \varrho, a))$ Refer to Fig. 3(a-c) Input: a frame P, an orientation $\varrho \in\{-1,+1\}$, and a convex vertex a of multiplicity 1 in $P$ such that $a b \in S$ and $b$ is not a vertex of P. Action: Let $c$ be the neighbor of a in polygon $P$ in orientation $\varrho$ (where ccw=1, $c w=-1)$. Replace the edge ac of $P$ with the polygonal path $a b+\operatorname{carc}(b, a, c)$.

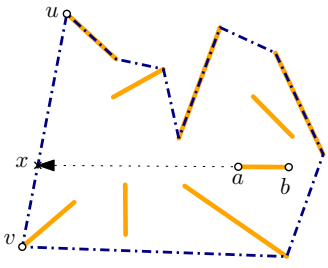

(a)

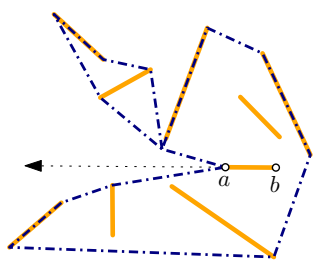

(b)

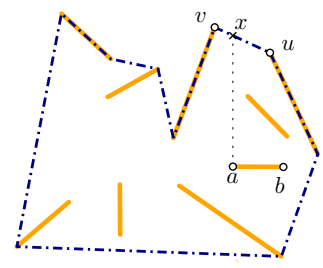

(c)

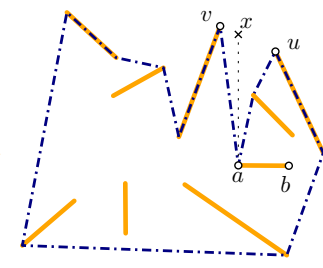

(d)

Figure 4 (a) A frame. (b) The result of operation $\operatorname{Dip}(P, a, b)$. (c) A frame. (d) The result of operation ShearDip $(P, a, x)$.

- Operation 3. (Dip $(P, a, b))$ Refer to Fig. 4(a-b). Input: a frame P, a segment ab $\in S$ such that neither a nor $b$ is a vertex of $P$, and the ray $\overrightarrow{b a}$ hits an edge of $P$ that is not a segment in S. Action: Assume that $\overrightarrow{b a}$ hits edge uv of $P$ at the point $x$. Replace the edge uv of $P$ with the polygonal path $\operatorname{carc}(u, x, a)+\operatorname{carc}(a, x, v)$. 
- Operation 4. (ShearDip(P,a,x)) Refer to Fig. 4(c-d). Input: a frame P, a segment endpoint $a$ in the interior of $P$, and a point $x$ in the interior of an edge of $P$ that is not a segment in $S$ such that ax does not cross $P$ or any segment in $S$. Action: Let $u v$ be the edge of $P$ that contains $x$. Replace the edge uv of $P$ with the polygonal path $\operatorname{carc}(u, x, a)+\operatorname{carc}(a, x, v)$.

Operations 1 and 2 have been previously used in [8, 13]; it was shown that if all reflex vertices in $P$ have been created by BuildCap operations, then property (F5) automatically holds [8, Sec. 2]. It is not difficult to see that if all reflex vertices in $P$ have been created by Dip operations, then property (F5) holds. However, this property does not extend to a mixed sequence of BuildCap and Dip operations, and certainly not for ShearDip operations. We maintain property (F5) by a careful application of these operations, using the fact that each set $Q_{i}(i=1, \ldots, r)$ is stabbed by a vertical line.

Note that Operations 1-4 can only increase the vertex set of the frame (the shortcut operation decreases the multiplicity of repeated vertices from 2 to 1 , but maintains the same vertex set). Initially, $P=\partial \operatorname{conv}(S)$, and so all vertices of conv $(S)$ remain vertices in $P$ in our algorithm. In particular, the leftmost and rightmost segment endpoint in $S$ are always vertices in $P$ (with multiplicity 1 by property (F4)). These vertices subdivide $P$ into an upper arc and a lower arc. As a convention, the leftmost (resp., rightmost) vertex is part of the lower arc (upper arc). We define an orientation for every vertex $v$ in a frame $P$ : If $v$ is in the lower arc and the left endpoint of a segment in $S$, or if it is in the upper arc the right endpoint of a segment in $S$, then $\varphi(v)=1$; otherwise $\varphi(v)=-1$. When our algorithm invokes the BuildCap operation at a vertex $v$, we use $\operatorname{BuildCap}(P, \varrho(v), v)$.

We now can justify the distinction between safe and unsafe reflex vertices.

- Lemma 3. Let $v$ be a reflex vertex of multiplicity 1 in a frame $P$ such that $v$ is safe. Then after any sequence of the above four operations, the multiplicity of $v$ remains 1.

Proof. Each operation creates at most one new reflex vertex, which has multiplicity 1; and possibly many convex vertices along the convex arcs, which may have multiplicity 1 or 2 . However, each point can be an interior vertex of at most one convex arc. Consequently, the multiplicity of a vertex $v$ can possibly increase from 1 to 2 if it is first a reflex vertex of multiplicity 1, and then visited for a second time (by a convex arc) as a convex vertex; see Fig. 3(c) and Fig. 4(d) for examples. If $v$ is a safe reflex vertex, then it cannot be an interior vertex of a convex arc, and so its multiplicity cannot increase from 1 to 2 .

Phase 1: Left Endpoints. Initially, the frame $P$ is the boundary of the convex hull $\operatorname{conv}(S)$. In the first phase of our algorithm, we use operations BuildCap and Dip as follows:

1. Let $P=\partial \operatorname{conv}(S)$.

2. While condition (a) or (b) below is applicable, do:

a. If there exits a segment $a b \in S$ such that the left endpoint $a$ is a vertex of $P$, but the right endpoint is not, then set $P:=\operatorname{BuildCap}(P, \varrho(a), a)$.

b. Else if there exists a segment $a b \in Q_{i}$ for some $i \in\{1, \ldots, r\}$ such that $a$ is the left endpoint, $a$ lies in the interior of $P$, and $\overrightarrow{b a}$ hits an edge $u v$ where $u v \notin S$, and the left endpoint of $u v$ is an endpoint of some segment in $Q_{i}$, then set $P:=\operatorname{Dip}(P, a, b)$.

3. Return $P$, and terminate Phase 1 .

An example is shown in Fig. 5. First we show that Phase 1 returns a frame.

- Lemma 4. All operations in Phase 2 maintain properties (F1)-(F6) for P. 


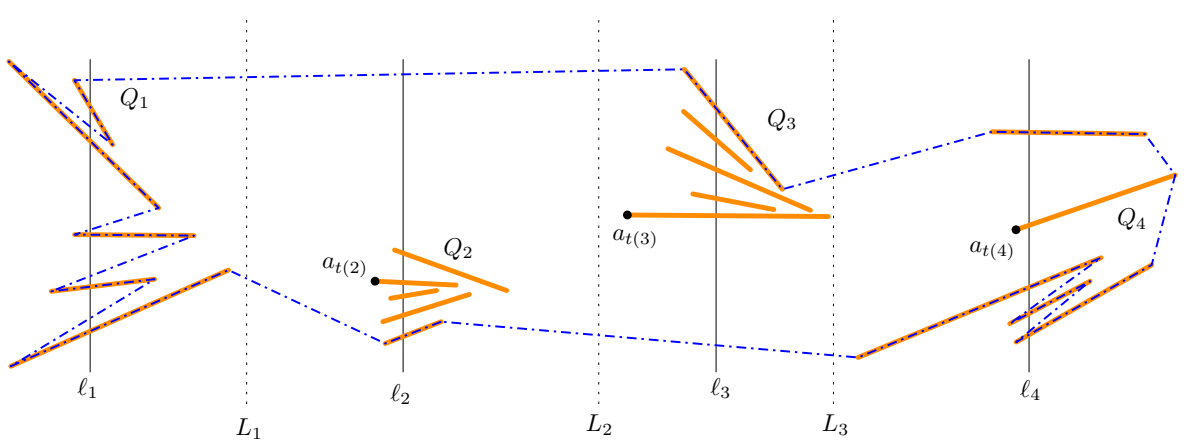

Figure 5 A set $\widehat{S}_{1}$ of 25 line segments; and the frame $P$ at the end of Phase 1.

We also make simple observation about the frame at the end of Phase 1:

- Lemma 5. Let $P$ be the frame returned by Phase 1 , and let $a b \in \widehat{S}_{1}$. If the left endpoint of $a b$ is a vertex of $P$, then it is a convex vertex of multiplicity 1.

The next lemma helps identify the segments in $Q_{i}$ whose left endpoints are not in $P$.

- Lemma 6. Let $P$ be the frame returned by Phase 1. Let $i \in\{1, \ldots, r\}$, and let $Q_{i}=$ $\left\{a_{j} b_{j}: j=1, \ldots,\left|Q_{i}\right|\right\}$, be sorted in increasing order by the $y$-coordinates of $a_{j} b_{j} \cap \ell_{i}$. If $a_{j}$ is a vertex in the lower (resp., upper) arc of $P$, then so is $a_{j^{\prime}}$ for all $j^{\prime}<j$ (resp., $j^{\prime}>j$ ).

By Lemma 6, the line segments in $\widehat{S}_{1}$ whose left endpoints are not in $P$ form a continuous interval. That is, for every $i \in\{1, \ldots, r\}$, there is a set of consecutive indices $M_{i} \subseteq$ $\left\{1, \ldots,\left|Q_{i}\right|\right\}$ (possibly $M_{i}=\emptyset$ or $M_{i}=\left\{1, \ldots,\left|Q_{i}\right|\right\}$ ) such that $j \in M_{i}$ if and only if the left endpoint of $a_{j} b_{j}$ is not in $P$. Let $Q_{i}^{\prime}=\left\{a_{j} b_{j}: j \in M_{i}\right\}$ and $S^{\prime}=\bigcup_{i=1}^{r} S_{i}^{\prime}$

Phase 2: Middle Segments. In Phase 2, we use ShearDip and Dip operations to reach the left endpoints of at least half of the segments in $S^{\prime}$, followed by BuildCap operation to reach the right endpoints of those segments if necessary. For $i=1, \ldots, r$, let $a_{t(i)}$ be the leftmost left endpoint in the set $S_{i}^{\prime}$. From some suitable point $x_{i}$ on the upper or the lower arc of $P$, we use $\operatorname{ShearDip}\left(P, a_{t(i)}, x_{i}\right)$ to reach $a_{t(i)}$. Choose the points $x_{i}, i=1, \ldots, r$ on the same (upper or lower) arc of $P$ by comparing the number of segments in $S_{i}^{\prime}$ above and below $a_{t(i)} b_{t(i)}$ for $i=1, \ldots, r$. The set of segments above and below are $A$ and $B$, respectively, defined as follows:

$$
\begin{aligned}
& A=\bigcup_{i=1}^{r} A_{i}, \text { where } A_{i}=\left\{a_{j} b_{j}: j \geq \ell(i), j \in M_{i},\right\}, \\
& B=\bigcup_{i=1}^{r} B_{i}, \text { where } B_{i}=\left\{a_{j} b_{j}: j \leq \ell(i), j \in M_{i},\right\} .
\end{aligned}
$$

If $|A| \geq|B|$, then we reach the vertices $a_{t(i)}$, for $i=1, \ldots, r$, from the upper arc; otherwise we reach them from the lower arc. Without loss of generality, assume that $|A| \geq|B|$.

It remains to specify the points $x_{i}$ for the operations $\operatorname{ShearDip}\left(P, a_{t(i)}, x_{i}\right)$. Consider the vertical upward ray from $a_{t(i)}$, and let $u_{i}$ be the first point on the ray that lies in the upper arc of $P$ or on a segment in $S$. If $u_{i}$ is in an edge of the upper arc, but not in a segment in $S$, then let $x_{i}:=u_{i}$. Otherwise, $u_{i}$ lies in some segment $a b \in Q_{i}$, which is either an edge or an internal diagonal of $P$. Since $a_{t(i)}$ is the leftmost left endpoint of a segment in $Q_{i}$ that 
is not a vertex in $P$, we know that the triangle $\Delta\left(a_{t(i)} u_{i} a\right)$ is empty, and in particular $a_{t(i)}$ sees vertex $a$. Furthermore, $a$ is a convex vertex of $P$ of multiplicity 1 (cf. Lemma 5 ). In this case, let $x_{i}$ be an interior point of edge $a_{0} a$. Phase 2 proceeds as follows:

1. For $i=1$ to $r$ :

If $M_{i} \neq \emptyset$, then set $P:=\operatorname{ShearDip}\left(P, a_{t(i)}, x_{i}\right)$.

2. While condition (a) or (b) below is applicable, do:

a. If there exits a segment $a b \in S$ such that the left endpoint $a$ is a vertex of $P$, but the right endpoint is not, then set $P:=\operatorname{BuildCap}(P, \varrho(a), a)$.

b. Else if there exists a segment $a b \in Q_{i}$ for some $i \in\{1, \ldots, r\}$ such that $a b$ lies in the interior of $P, a$ is the left endpoint, and $\overrightarrow{b a}$ hits an edge $u v$ where $u v \notin S$, but both $u$ and $v$ are endpoints of some segments in $Q_{j}, j \geq i$, then set $P:=\operatorname{Dip}(P, a, b)$.

3. Return $P$, and terminate Phase 2 .

- Lemma 7. All operations in Phase 2 maintain properties (F1)-(F6) for P; and at the end of Phase 2, every vertex $a_{t(i)}, i=1, \ldots, r$, has multiplicity 1 in $P$.

- Lemma 8. At the end of Phase 2, $P$ visits both endpoints of all segments in A. Consequently, $P$ visits both endpoints of at least half of the segments in $\widehat{S}_{1}$.

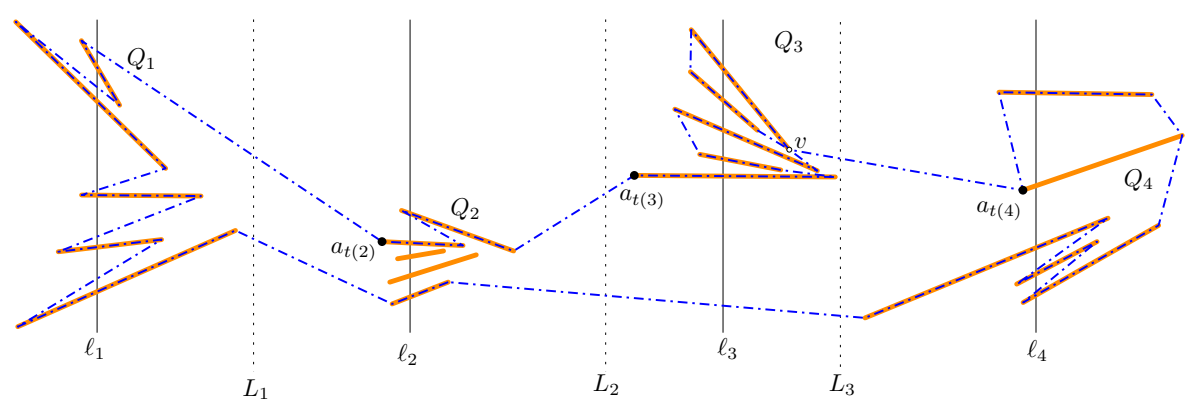

Figure 6 The set $\widehat{S}_{1}$ from Fig. 5, and frame $P$ at the end of Phase 2. Vertex $v$ has multiplicity 2 .

Phase 3: Right Endpoints. At the end of Phase 2, $P$ visits the left endpoint of every segment in $A$, and none in $B \backslash A$. However, it may visit the right endpoint of some segments in $B \backslash A$. In this phase, we use BuildCap operations to ensure that $P$ visits both endpoints of these segments. Phase 3 proceeds as follows.

1. While condition (a) below is applicable, do

a. If there exists a segment $a b \in S$ such that the one endpoint, say $b$, is a vertex of $P$, but the other endpoint is not, then set $P:=\operatorname{BuildCap}(P, \varrho(b), b)$.

2. Return $P$, and terminate Phase 3 .

At the end of Phase 3, we obtain a frame $P$ that contains, for each segment, either both endpoints or neither endpoint; see Fig. 6. Some vertices may have multiplicity 2, but the multiplicity of the special vertices $a_{t(i)}(i=1, \ldots, r)$ remains 1 .

- Lemma 9. All operations in Phase 3 maintain properties (F1)-(F6) for $P$; and the multiplicity of every vertex $a_{t(i)}, i=1, \ldots, r$, remains 1 .

- Lemma 10. Let $P$ be the frame at the end of Phase 3. If one endpoint of a segment in $\widehat{S}_{1}$ is a vertex in $P$, then so is the other endpoint. 
Phase 4: Obtaining a Simple Polygon. In the last phase of our algorithm, we set $P=$ ChopWedges $(P)$. This is a valid operation by property (F5). The resulting frame $P$ is a simple polygon whose vertex set is the same as at the end of Phase 3. By Lemma 10, if one endpoint of a segment in $\widehat{S}_{1}$ is a vertex in $P$, then so is the other endpoint. Consequently, $P$ is a circumscribing polygon for a set of segments in $\widehat{S}_{1}$, which we denote by $\widehat{S}_{2}$. By Lemma 8 , we have $\left|\widehat{S}_{2}\right| \geq\left|\widehat{S}_{1}\right| / 2$, as claimed. This completes the proof of Theorem 1 .

\section{Disjoint Segments versus Disjoint Rays}

In this section, we give two sufficient conditions for an arrangement of disjoint segments to admit a circumscribing polygon. Both conditions involve extending the segments.
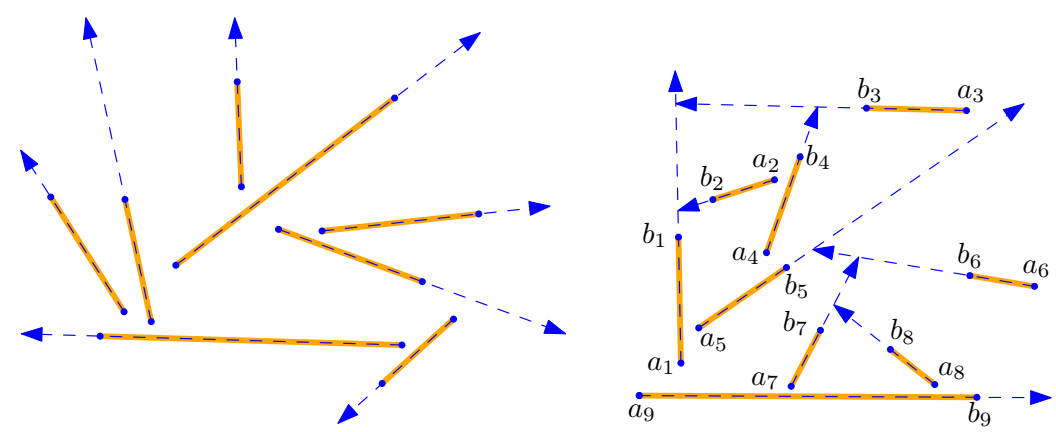

Figure 7 Left: an arrangement of disjoint segments extensible to rays. Right: an arrangement of disjoint segments that is not extensible to rays, but admits escape routes.

(C1) A set $S$ of $n$ disjoint line segments is extensible to rays if there exists a set $R$ of $n$ disjoint rays, each of which contains a segment from $S$; see Fig. 7(left).

(C2) A set $S$ of $n$ disjoint line segments admits escape routes if there exists an ordering and orientation of the segments $S=\left\{a_{i}, b_{i}: i=1, \ldots, n\right\}$ if the following process produces a set of $n$ rays or directed segments that do not cross any segment in $S$ : For $i=1, \ldots, n$, shoot a ray from $b_{i}$ in direction $\overrightarrow{a_{i} b_{i}}$ that ends at the first point where it hits a previous ray or goes to infinity; see Fig. 7 (right).

Clearly, (C1) implies (C2), but the converse is false in general. We can test property (C1) in $O(n \log n)$ time. Indeed, there are two possible directions to extend each segment into a ray, which can be encoded by a Boolean variable, and pairwise disjointness can be expressed by a 2SAT formula. For given ordering and orientation, it takes $O\left(n \log ^{2} n\right)$ time to test whether the extensions form escape routes [11]. However, we do not know whether condition (C2) can be tested efficiently. Here we show that (C1) and (C2) each imply the existence of a circumscribing polygon.

Theorem 11. If $S$ is a set of disjoint line segments satisfying (C2), then there is a circumscribing polygon for $S$.

Proof sketch. By $(\mathrm{C} 2)$, we may assume that $S=\left\{a_{i} b_{i}: i=1, \ldots n\right\}$ such that if we shoot a ray from $b_{i}$ in direction $\overrightarrow{a_{i} b_{i}}$ for $i=1, \ldots, n$, then each ray either goes to infinity or intersects a previous ray. We call the part of the ray $\overrightarrow{a_{i} b_{i}}$ from $b_{i}$ to the first point where it intersects a previous ray or $\partial \operatorname{conv}(S)$ the extension of segment $a_{i} b_{i}$.

Given the ordering and orientation of the segments in $S$, we construct a circumscribing polygon using the following algorithm, using the operations BuildCap and Dip introduced in Section 2; see Fig. 8 for an example. 


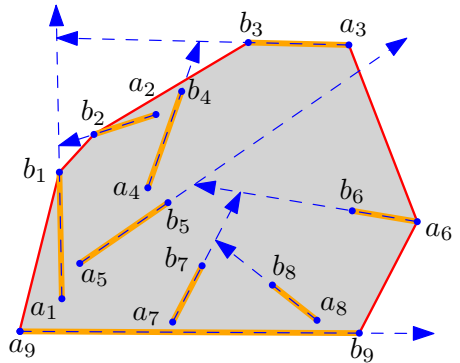

(a)

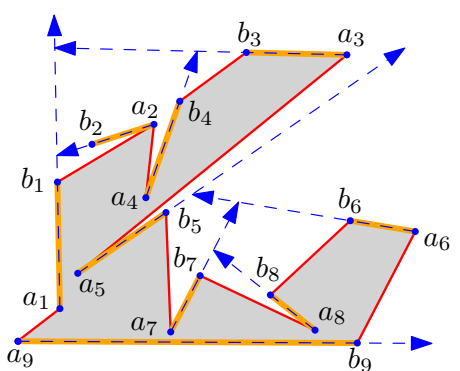

(c)

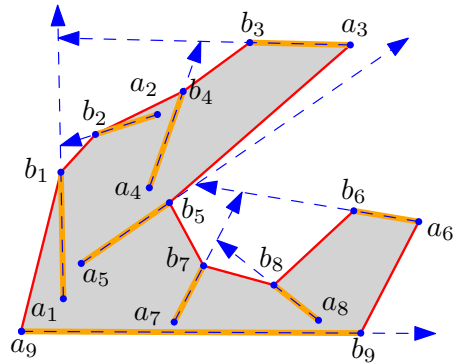

(b)

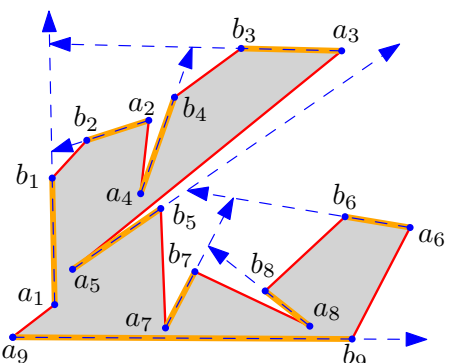

(d)

Figure 8 (a) An arrangement of 9 segments that admit escape routes from Fig. 7(right), and $P=\operatorname{conv}(S)$. (b) Polygon $P$ after the for loop of Dip operations. (c) Polygon $P$ after the for loop of BuildCap operations. (d) The circumscribing polygon for $S$ after the ChopWedges operation.

1. Initialize $P:=\partial \operatorname{conv}(S)$.

2. For $i=1$ to $n$ : if $b_{i}$ is not a vertex of $P$, then set $P:=\operatorname{Dip}\left(P, b_{i}, a_{i}\right)$.

3. For $i=1$ to $n$ : if $a_{i}$ is not a vertex of $P$, then set $P:=\operatorname{BuildCap}\left(P, 1, b_{i}\right)$.

4. ChopWedges $(P)$.

5. Return $P$.

The above results link the circumscribing polygon problem to the problem of extending line segments to rays. We now give an upper bound for the latter problem that seems to imply that the lower bound of the former problem (Theorem 1) is tight.

Lemma 12. For every $n \in \mathbb{N}$, there is a set $S$ of $n$ disjoint line segments in the plane such that the cardinality of every subset $S^{\prime} \subseteq S$ that admits an escape route is $\left|S^{\prime}\right| \leq 2\lceil\sqrt{n}\rceil-1$.

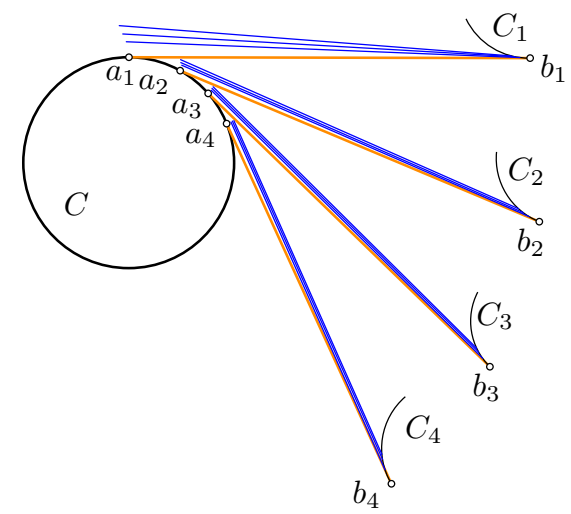

Figure 9 Our lower bound construction for $k=4$. 
Proof sketch. We create a problem instance $S$ with $\sqrt{n}$ groups, each containing $\sqrt{n}$ segments (see Fig. 9). The key idea is that in any subset $Q$ of $S$ that admits escape routes, only segments of one group can extend to the left. Moreover, we show that from each group only one segment can extend to the right, giving the $2\lceil\sqrt{n}\rceil-1$ bound.

\section{$4 \quad$ Hardness for Circumscribing Polygons}

In this section we prove that it is NP-hard to decide whether a given set of disjoint line segments admits a circumscribing polygon. We reduce from a problem that we call HAMILTOnian Path in 3-Connected Planar Cubic Graphs with Start Edge (HP3CPG-SE): Given a 3-connected cubic planar graph $G=(V, E)$ and an edge $u v \in E$, decide whether $G$ has Hamiltonian path whose first edge is $u v$, which is NP-complete [7, p. 713].

- Theorem 13. It is NP-complete to decide whether a given PSLG admits a circumscribing polygon, even if the PSLG is max-degree-2.

Proof sketch. The membership to NP is trivial. Let $G=(V, E)$ and $u v \in E$ be an instance of HP3CPG-SE, and let $n=|V|$ (Fig. 10 (a)). We modify $G$ by a $Y \Delta$-transform at $u$ producing a graph $G^{\prime}$ with a new triangular face $\Delta\left(a b u^{\prime}\right)$ (Fig. 10 (b)). It is clear that $G$ admits a Hamiltonian path starting with $u v$ if and only if $G^{\prime}$ admits a Hamiltonian path starting with $a b u^{\prime} v$. Embed $G^{\prime}$ so that the outer face is $\Delta\left(a b u^{\prime}\right)$ (Fig. 10 (c)). Delete the edge incident to $a(b)$ that does not bound the outer face. Rotate by a small amount all edges that do not bound the outer face, splitting each internal degree- 3 vertex $w$ into a triangle $\Delta\left(w_{1} w_{2} w_{3}\right)$ (Fig. 10(d) and Fig. 11(a-b)). We call these newly created triangles transparent faces, and every remaining internal face opaque. Create a small new edge in each transparent face, and shrink each opaque face by a small amount using its straight skeleton (Fig. 10 (e)). After this step, each added small segment can still only see the three vertices (Fig. 11 (c)). This construction defines a max-degree-2 PSLG $\widehat{G}=(\widehat{V}, \widehat{E})$.

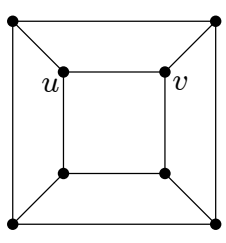

(a)

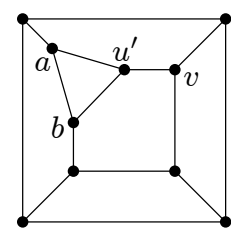

(b)

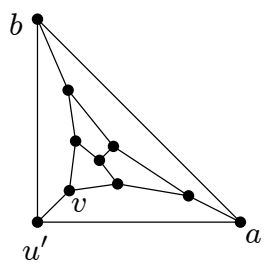

(c)

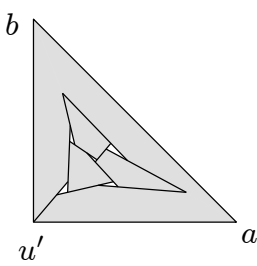

(d)

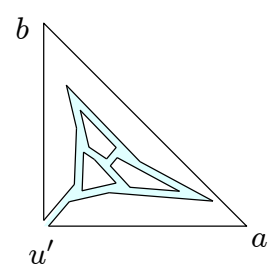

(e)

Figure 10 (a) A 3-connected cubic graph $G$ with special edge $u v$. (b) Graph $G^{\prime}$ after a $Y \Delta$ transform around vertex $u$. (c) A convex embedding of $G^{\prime}$ such that the outer face is $\Delta_{u}$. (d) Rotating the supporting line of internal edges. (e) Thickening the edges into corridors.

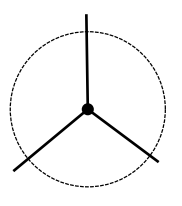

(a)

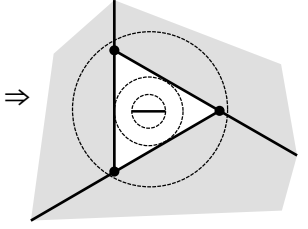

(b)

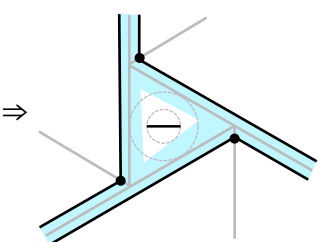

(c)

Figure 11 A chamber incident to three corridors and a segment in the chamber that sees only three other vertices. 
A circumscribing polygon of $\widehat{G}$ must enclose all of its opaque faces. We partition the regions of the outer face of $\widehat{G}$ that lies in the convex hull of the embedding into corridors and chambers. They correspond to edges and vertices of $G^{\prime}$ respectively. We show that a circumscribing polygon of $\widehat{G}$ must behave as in Figs. $12(\mathrm{a}-\mathrm{d})$ in the vicinity of the endpoints of a corridor, up to symmetry. Then, such a circumscribing polygon defines a Hamiltonian path on the chambers. Hence, $\widehat{G}$ admits a circumscribing polygon if and only if $G$ admits a Hamiltonian path starting with $u v$.

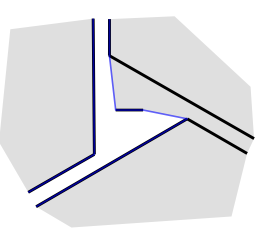

(a)

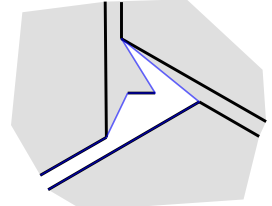

(b)

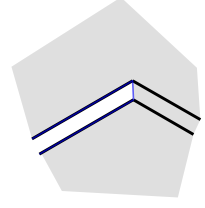

(c)

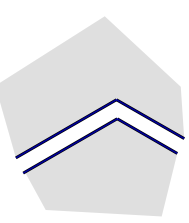

(d)

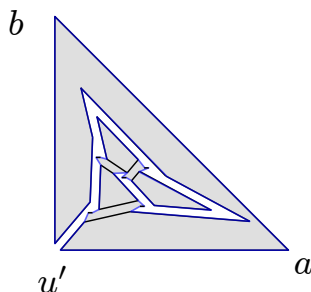

(e)

Figure 12 A circumscribing polygon $\widehat{P}$ at a chamber and a solution to the reduction in Fig. 10 .

\section{Simple Polygonizations of Disjoint Segments}

Rappaport [18] proved that it is NP-hard to decide whether a given PSLG $G=(V, E)$ admits a polygonization. The reduction in [18] is from Hamiltonian Path in Planar CubiC GraPhs (HPPCG) and produces an instance in which $G$ is a union of disjoint paths, every edge in $E$ is horizontal or vertical, and the vertices in $V$ have integer coordinates, bounded by a polynomial in $n=|V|$. In this section, we describe the connection gadget made of disjoint line segments that simulates a pair of line segments that share an endpoint. Using this gadget, we show that finding a simple polygonization of disjoint line segments is NP-hard. Informal description of the connection gadget. Refer to Figure 13. Given a PSLG $G=(V, E)$, with a vertex $p_{2} \in V$ of degree 2 , incident to $p_{1} p_{2}, p_{2} p_{3} \in E$, delete the edge $p_{1} p_{2}$, and insert 6 new edges $p_{1} p_{2}^{\prime}, p_{4} p_{5}, p_{6} p_{7}, p_{8} p_{9}, p_{10} p_{11}, p_{12} p_{13}$, and 11 new vertices $p_{2}^{\prime}$ and $p_{i}(i=3, \ldots, 13)$. Denote by $G^{\prime}=\left(V^{\prime}, E^{\prime}\right)$ the resulting new PSLG. We choose the position of the new vertices close to $p_{2}$ so that: (i) the two small segments $p_{6} p_{7}$ and $p_{10} p_{11}$ are only visible from points $p_{4}, p_{5}, p_{8}$, and $p_{9}, p_{12}, p_{13}$ respectively; (ii) the union of the visibility regions of $p_{4}, p_{5}, p_{12}$, and $p_{13}$ contain only vertices $p_{2}, p_{2}^{\prime}, p_{4}, \ldots, p_{13}$ and no other vertices.

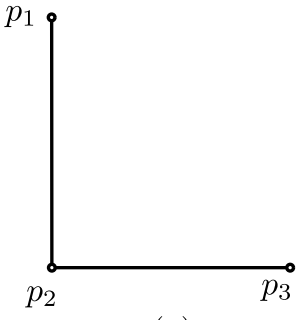

(a)

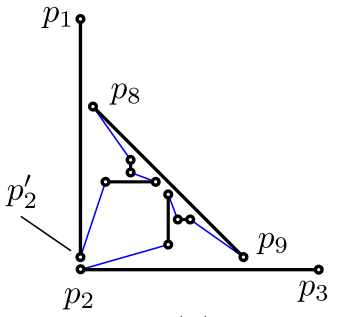

(b)

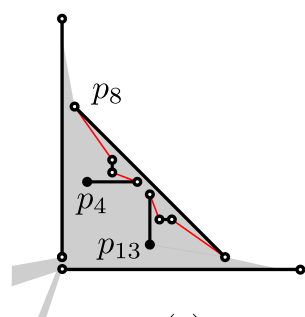

(c)

Figure 13 (a) Two line segments $p_{1} p_{2}$ and $p_{2} p_{3}$. (b) Connection gadget that simulates (a) using seven disjoint line segments. The polygonal path shown with black and blue line segments is $\left[p_{1}, p_{2}^{\prime}, p_{4}, p_{5}, p_{6}, p_{7}, p_{8}, p_{9}, p_{10}, p_{11}, p_{12}, p_{13}, p_{2}, p_{3}\right]$. (c) The union of the visibility region of the solid black points $p_{4}$ and $p_{13}$. 
- Theorem 14. It is NP-complete to decide whether a set $S$ of disjoint line segments admits a simple polygonization, even if $S$ contains only segments with 4 distinct slopes.

Proof. Membership in NP is proven in [18]. We reduce NP-hardness from finding polygonizations for a disjoint union of paths. Let $G=(V, E)$ be a PSLG produced by the reduction in [18], and let $n=|V|$. We modify $G$ by simultaneously replacing every vertex of degree 2 by a connection gadget (described above), and show that the resulting planar straight-line matching $M$ admits a polygonization if and only if $G$ does. Since each gadget is constructed independently, all coordinates can be described by polynomials as they are each obtained by a constant number of intersections between lines and circles determined by $G$. Since $G$ contains only axis-parallel edges, edges of $M$ have up to four distinct slopes. The reduction runs in polynomial time.

We now show that $M$ admits a polygonization if and only if $G$ does. Note that the connection gadget places edges in the convex corner of a degree- 2 vertex in $G$, and it does not block or create visibility between two leafs of $G$. By construction, if $p$ is a leaf in $G$, then the set of other leaves visible from $p$ remains same in $M$. Since $G$ is max-degree-2, it remains to prove that, for every connection gadget, a polygonization of $M$ must contain a chain of length 11 from $p_{2}^{\prime}$ to $p_{2}$ that uses only edges of the connection gadget.

By property (i) of the connection gadget, if a simple polygonization $P$ of $M$ exists, $P$ must connect $p_{8}$ with $p_{6}$ or $p_{7}$, and $p_{6}$ or $p_{7}$ to $p_{4}$ or $p_{5}$, otherwise $P$ would contain a cycle of length 4 and $P$ would be disconnected. The same argument applies to vertices $p_{9}, \ldots, p_{13}$. Fig. 13(c) shows the forced edges in a polygonization in red. By property (ii), $p_{2}^{\prime}$ must be adjacent to $p_{4}$ or $p_{5}$, and $p_{2}$ must be adjacent to $p_{12}$ or $p_{13}$, or else either $P$ would contain a cycle of length 10 and $P$ would be disconnected, or $P$ would not be simple.

\section{Conclusions}

Our results raise interesting open problems, among others, about circumscribing polygons in the plane (Section 6.1), and about higher dimensional generalizations (Section 6.2).

\subsection{Geometric Matching or Few Slopes}

As noted above, Urabe and Watanabe [21] constructed an arrangement of 16 disjoint segments in $\mathbb{R}^{2}$ that does not admit a circumscribing polygon. If all segments have the same slope (but they are not all collinear), then there always exists a circumscribing polygon. We conjecture that disjoint segments with two distinct slopes still admit a circumscribing polygon. Here we present negative instances with three slopes.

- Proposition 15. For every $n \geq 9$, there is a set of $n$ disjoint segments of 3 different slopes that do not admit a circumscribing polygon.

Our construction for $n=9$ is depicted in Figure 14(a).

\subsection{Higher Dimensions}

Generalizations to higher dimensions are also of interest. For a set $V$ of points in $\mathbb{R}^{3}$, a polyhedralization is a polyhedron homotopic to a sphere whose vertex set is $V^{1}$. It is known that every set of $n \geq 4$ points in general position admits a polyhedralization [1], and even a polyhedralization of bounded vertex degree [3].

1 We thank Joe Mitchell for introducing us to the high dimensional variations of this problem. 


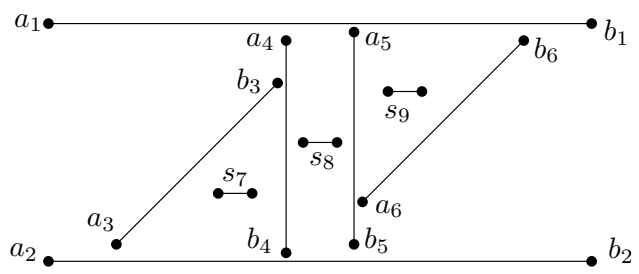

(a)

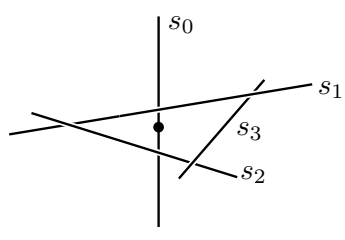

(b)

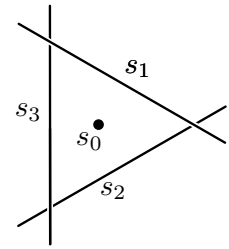

(c)

Figure 14 (a) A set of 9 disjoint line segments of slopes 0 , 1, and $\infty$, that do not admit a circumscribing polygon. (b-c) A set of 4 disjoint segments in $\mathbb{R}^{3}$ that do not admit a polyhedralization: perspective view (b) and view from above (c).

For a set $S$ of disjoint line segments in $\mathbb{R}^{3}$, we define a polyhedralization as a polyhedron homotopic to a ball whose vertices are the segment endpoints, and every segment in $S$ is either an edge or an (external or internal) diagonal. A polyhedralization circumscribes $S$ if every segment in $S$ is an edge or an internal diagonal. It is not difficult to see that an arrangement of disjoint segments in general position in $\mathbb{R}^{3}$ need not admit a polyhedralization.

- Proposition 16. For every $n \geq 4$, there is a set of $n$ disjoint segments in $\mathbb{R}^{3}$ that do not admit a polyhedralization.

Our construction is depicted in Figure 14(b-c).

We suspect that it is NP-hard to decide whether a set of $n$ segments in $\mathbb{R}^{3}$ admit a polyhedralization (or even a circumscribing polyhedralization). However, our proof techniques do not seem to extend to higher dimensions.

\subsection{Open Problems}

We conclude with a collection of open problems.

1. In Section 5 we established NP-hardness for the polygonization problem, even when the input consists of disjoint segments with four distinct slopes. Is it NP-hard to decide whether $n$ disjoint axis-parallel segments in the plane admit a polygonization? Is it NP-hard for segments of 3 possible directions?

2. In Section 4 we proved that it is NP-complete to decide whether a 2-regular PSLG admits a circumscribing polygon. We do not know whether the problem remains hard for 1-regular PSLGs (i.e., disjoint line segments). The connection gadgets we designed for the polygonization problem (Section 4) do not seem to work for circumscribing polygons. Is it NP-hard to decide whether $n$ disjoint segments admit a circumscribing polygon?

3. Does every arrangement of disjoint axis-parallel segments in $\mathbb{R}^{2}$, not all in a line, admit a circumscribing polygon?

4. Does every arrangement of disjoint line segments in $\mathbb{R}^{3}$, not all in a plane, admit a circumscribing polyhedron?

5. We can decide in $O(n \log n)$ time whether $n$ disjoint segments are extensible to disjoint rays (Section 3). Can we decide efficiently whether they admit escape routes?

6. Let $f(n)$ be the maximum integer such that every set of $n$ disjoint segments contains $f(n)$ segments that admit a circumscribing polygon. In Section 2 , we prove a lower bound of $f(n)=\Omega(\sqrt{n})$. Is it possible that $f(n)=\Omega(n)$ ? Is there a nontrivial upper bound? 
7. Let $g(n)$ be the maximum integer such that every set of $n$ disjoint segments contains $g(n)$ segments that are extensible to disjoint rays. Theorem 11 implies $g(n) \leq f(n)$. PA Ramsey-type result on the intersection graph of rays [12, Remark 2] yields $g(n)=\Omega\left(n^{1 / 3}\right)$, and Lemma 12 gives $g(n)=O(\sqrt{n})$. What is the asymptotic growth rate of $g(n)$ ?

8. Let $h(n)$ be the maximum integer such that every set of $n$ disjoint segments contains $h(n)$ segments that admit an escape route. Theorem 11 implies $g(n) \leq h(n) \leq f(n)$. We have $h(n)=\Omega\left(n^{1 / 3}\right)$ and $h(n)=O(\sqrt{n})$. What is the asymptotic growth rate of $h(n)$ ?

\section{References}

1 Pankaj K. Agarwal, Ferran Hurtado, Godfried T. Toussaint, and Joan Trias. On polyhedra induced by point sets in space. Discrete Applied Mathematics, 156(1):42-54, 2008. doi: $10.1016 / \mathrm{j}$.dam.2007.08.033.

2 Hugo A. Akitaya, Matias Korman, Mikhail Rudoy, Diane L. Souvaine, and Csaba D. Tóth. Circumscribing Polygons and Polygonizations. CoRR, abs/1903.07019, 2019. arXiv:1903. 07019.

3 Gill Barequet, Nadia Benbernou, David Charlton, Erik D. Demaine, Martin L. Demaine, Mashhood Ishaque, Anna Lubiw, André Schulz, Diane L. Souvaine, Godfried T. Toussaint, and Andrew Winslow. Bounded-degree polyhedronization of point sets. Comput. Geom., 46(2):148-153, 2013. doi:10.1016/j.comgeo.2012.02.008.

4 Norishige Chiba and Takao Nishizeki. The Hamiltonian cycle problem is linear-time solvable for 4-connected planar graphs. Journal of Algorithms, 10(2):187-211, 1989. doi:10.1016/ 0196-6774(89) 90012-6.

5 Emilio Di Giacomo and Giuseppe Liotta. The Hamiltonian Augmentation Problem and Its Applications to Graph Drawing. In Md. Saidur Rahman and Satoshi Fujita, editors, Proc. 4 th International Workshop Algorithms and Computation (WALCOM), volume 5942 of LNCS, pages 35-46, Berling, 2010. Springer. doi:10.1007/978-3-642-11440-3_4.

6 Alfredo García, Marc Noy, and Javier Tejel. Lower bounds on the number of crossing-free subgraphs of $\mathrm{K}_{\mathrm{N}}$. Comput. Geom., 16(4):211-221, 2000. doi:10.1016/S0925-7721(00) 00010-9.

7 Michael R. Garey, David S. Johnson, and Robert E. Tarjan. The Planar Hamiltonian Circuit Problem is NP-Complete. SIAM J. Comput., 5(4):704-714, 1976. doi:10.1137/0205049.

8 Michael Hoffmann and Csaba D. Tóth. Segment endpoint visibility graphs are Hamiltonian. Comput. Geom., 26(1):47-68, 2003. doi:10.1016/S0925-7721(02)00172-4.

9 Ferran Hurtado and Csaba D. Tóth. Plane Geometric Graph Augmentation: A Generic Perspective. In János Pach, editor, Thirty Essays on Geometric Graph Theory, pages 327-354. Springer, New York, 2013. doi:10.1007/978-1-4614-0110-0_17.

10 Mashhood Ishaque, Diane L. Souvaine, and Csaba D. Tóth. Disjoint Compatible Geometric Matchings. Discrete ES Computational Geometry, 49(1):89-131, 2013. doi:10.1007/ s00454-012-9466-9.

11 Mashhood Ishaque, Bettina Speckmann, and Csaba D. Tóth. Shooting Permanent Rays among Disjoint Polygons in the Plane. SIAM J. Comput., 41(4):1005-1027, 2012. doi: $10.1137 / 100804310$.

12 David Larman, Jiř́i Matoušek, János Pach, and Jenő Törőcsik. A Ramsey-Type Result for Convex Sets. Bull. London Math. Soc., 26(2):132-136, 1994. doi:10.1112/blms/26.2.132.

13 Andranik Mirzaian. Hamiltonian Triangulations and Circumscribing Polygons of Disjoint Line Segments. Comput. Geom., 2:15-30, 1992. doi:10.1016/0925-7721(92)90018-N.

14 Joseph O'Rourke and Jennifer Rippel. Two Segment Classes with Hamiltonian Visibility Graphs. Comput. Geom., 4:209-218, 1994. doi:10.1016/0925-7721(94)90019-1.

15 Kenta Ozeki, Nico Van Cleemput, and Carol T. Zamfirescu. Hamiltonian properties of polyhedra with few 3-cuts - a survey. Discrete Mathematics, 341(9):2646-2660, 2018. doi: $10.1016 / j . \operatorname{disc} .2018 .06 .015$. 
16 János Pach and Eduardo Rivera-Campo. On circumscribing polygons for line segments. Comput. Geom., 10(2):121-124, 1998. doi:10.1016/S0925-7721(97)00023-0.

17 János Pach and Rephael Wenger. Embedding Planar Graphs at Fixed Vertex Locations. Graphs and Combinatorics, 17(4):717-728, 2001. doi:10.1007/PL00007258.

18 David Rappaport. Computing simple circuits from a set of line segments is NP-complete. SIAM Journal on Computing, 18(6):1128-1139, 1989. doi:10.1137/0218075.

19 David Rappaport, Hiroshi Imai, and Godfried T. Toussaint. Computing simple circuits from a set of line segments. Discrete ES Computational Geometry, 5(3):289-304, 1990. doi: 10.1007/BF02187791.

20 Micha Sharir, Adam Sheffer, and Emo Welzl. Counting plane graphs: Perfect matchings, spanning cycles, and Kasteleyn's technique. J. Comb. Theory, Ser. A, 120(4):777-794, 2013. doi:10.1016/j.jcta.2013.01.002.

21 Masatsugu Urabe and Mamoru Watanabe. On a Counterexample to a Conjecture of Mirzaian. Comput. Geom., 2:51-53, 1992. doi:10.1016/0925-7721(92)90020-S.

22 Hassler Whitney. A Theorem on Graphs. Annals of Mathematics, 2nd Ser., 32(2):378-390, 1931. 